\title{
Safeguard research in the time of COVID-19
}

\author{
As the COVID-19 pandemic shuts down labs across the globe, funders and institutions must step up to support \\ scientists and ensure the healthy future of research.
}

$\mathrm{O}$ n 11 March, 2020, World Health Organization Director-General Tedros Adhanom Ghebreyesus declared COVID-19 a pandemic. The announcement prompted governments to intensify their regional- and country-level responses in the following weeks. In the absence of a vaccine or approved drugs against SARS-CoV-2, social distancing has been the only available approach to try to slow the spread of the virus. Entire countries have implemented aggressive lockdown strategies. Schools are closed, sporting events cancelled, public gatherings banned and a range of non-essential businesses shuttered indefinitely.

Worldwide, as universities and research centers have abruptly stopped their activities, many scientists justifiably worry about the effects of these shutdowns on their research and the future of science at large. Uncertainty around investigators' ability to support trainees, renew grants and fellowships, and continue career development is at an all-time high. The true impact of such a large-scale, indefinite cessation of research projects is unknown, but the suspension will certainly be long lasting and, in some cases, might be irreversibly damaging.

Biomedical scientists running wet labs have been particularly affected. Working at the bench is no longer an option and countless experiments have been terminated prematurely. In many institutions, laboratory and support-staff numbers have been pared down to only those performing essential functions. Clinical scientists have been mandated to temporarily cease research activities so that they can be redeployed into medical practice. And many clinical trials have been paused or terminated early to ensure patient safety. A majority of conferences, too, have been cancelled or postponed.

In the face of the challenges brought about by these unprecedented circumstances, the scientific community has responded with resilience, adaptation and ingenuity. Many universities and research institutes are acting to support staff, by facilitating flexible work-at-home arrangements for those who will have additional care responsibilities, as well as paid leave for custodial workers and others who may no longer be able to perform their usual duties. Researchers who are social distancing are finding new ways to connect and communicate. Last month, the president of the European Association for Cancer Research, Alberto Bardelli, provided an account of his own lab's closure in Nature. He talks of disappointment but also of staying connected virtually with online lab meetings, chat rooms and coffee breaks. Some conferences have gone virtual. The Conference on Retroviruses and Opportunistic Infections (CROI), for example, was scheduled to convene in Boston in early March. Just 2 days before the start of the event, CROI was reformatted into prerecorded webcasts. Adjustments were made during the conference to better enable discussions and questions on the presentations-a model that could be followed by others.

Some public and non-profit funders have been quick to respond to the rapidly changing landscape of the public-health emergency. For example, Wellcome, a research charity based in London, has issued guidance for grant holders that includes provisions for salary and sick leave for individuals who are ill, or who must selfisolate or care for someone sick, as well as an extension of grants to account for the suspension period. Although when this current situation will end is unknown, reopening laboratories and restarting experimental and clinical studies will inevitably require substantial additional funding. Given that many grant holders will not be spending their funds immediately on reagents and travel, grants with use-itor-lose-it clauses must be open to revision. All funding agencies must act now to plan for researchers' return to their labs to avoid unnecessary delays.

In the case of ongoing clinical trials, responsibility and care for participants must remain the priority. Although many clinical trials have temporarily suspended recruitment, there remains an ethical obligation to continue to offer potentially life-saving experimental therapies, for example, CAR T cell treatments, to those who need them. The National Institutes of Health (NIH) and the US Food and Drug Administration have issued comprehensive guidelines for maintaining good clinical practice and minimizing risks to trial integrity in these cases. The NIH guidelines also include provisions for the extension of grants and for additional funds for unanticipated costs incurred to protect patient safety during the COVID-19 pandemic; these provisions should be taken as a model to support clinical researchers whose work has been put on pause.

In this time of crisis, when economic uncertainty reigns, questioning why it is so crucial to protect and promote the continuity of the scientific enterprise is warranted. The answer lies in the commitment of researchers to use their collective wealth of knowledge and skill sets to contribute to ending the pandemic. Although governments have largely failed to provide enough tests to detect COVID19 in the population-an essential measure recommended by the World Health Organization-scientists are stepping in to assist the population by developing, scaling up and performing diagnostic tests. Reports of scientists donating lab reagents to clinical-testing sites are being shared daily on Twitter. Many researchers have refocused their work and are trying to develop ways to provide faster viral diagnosis, to understand the biology of the disease and to test whether existing drugs can be repurposed to treat patients with COVID-19. Many others are working to develop models to aid in understanding of how the pandemic is evolving, how to reduce its impact in areas not yet heavily affected by the virus and how to test whether measures of social distancing and self-quarantining are having the intended effects in 'flattening the curve'.

First responders, doctors, nurses and health professionals are at the frontlines of the fight against the virus-but scientists remain at the core of the solution. Governments, funders, universities and research institutes must support scientists through these troubling times. They must continue to adapt to the ongoing fast-paced changes in the research landscape and must be ready to support the scientific community and their research after COVID-19.

Published online: 2 April 2020 https://doi.org/10.1038/s41591-020-0852-1 